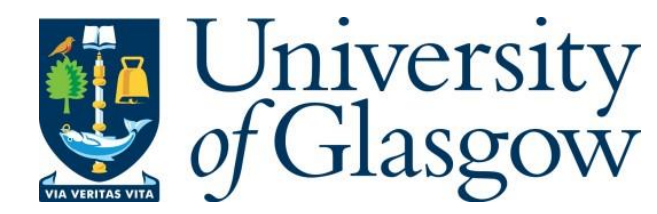

Héliot, F., Sambo, Y. A., and Imran, M. A. (2014) Energy-Efficient Interference-Aware Precoding for the Downlink of Multi-Cell Multi-User MIMO Systems. In: 2014 European Conference on Networks and Communications (EuCNC), Bologna, Italy, 2326 Jun 2014, ISBN 9781479952809.

There may be differences between this version and the published version. You are advised to consult the publisher's version if you wish to cite from it.

http://eprints.gla.ac.uk/136586/

Deposited on: 9 February 2017

Enlighten - Research publications by members of the University of Glasgow http://eprints.gla.ac.uk 


\title{
Energy-Efficient Interference-aware Precoding for the Downlink of Multi-cell Multi-user MIMO Systems
}

\author{
Fabien Héliot, Yusuf Sambo, and Muhammad Ali Imran \\ Centre for Communication Systems Research (CCSR), Faculty of Electronics \& Physical Sciences, \\ University of Surrey, Guildford GU2 7XH, UK. Email: F.Heliot@surrey.ac.uk
}

\begin{abstract}
Energy efficiency (EE) is fast becoming a key performance indicator for designing future wireless communication systems. Equally, precoding/power allocation has proved to be very effective for improving the spectral efficiency (SE) of multiuser (MU) multi-input multi-output (MIMO) communication systems. In a multi-cell environment, other-cell interference (OCI) degrades both the $\mathrm{SE}$ and $\mathrm{EE}$ performances of the system. We design here an energy efficient OCI-aware precoding/power allocation algorithm for the downlink of MU-MIMO systems by relying on regularized channel inversion and considering a realistic multi-antenna power consumption model. The performances of our proposed scheme are assessed both in presence and absence of OCI and results demonstrate the effectiveness of our approach for mitigating OCI. In addition, results show that our approach improves the EE of the system by saving transmit power in comparison with a traditional SE-based precoding/power allocation approach.

Index Terms-Energy efficiency, precoding, MIMO systems, multi-cell, realistic power model.
\end{abstract}

\section{INTRODUCTION}

The current increase in data transmitted over wireless networks has recently lead to a sharp increase in energy consumption and carbon emissions of such networks, which could jeopardize the economical and environmental sustainability of mobile communications. Thus, a paradigm shift towards energy-efficient networks is currently taking place in the ICT community. Indeed, the energy efficiency (EE), which is most commonly expressed in bit-per-Joule [1] or Joule-perbit [2], [3], is fast becoming a key performance indicator for future wireless communication systems, alongside the wellestablished spectral efficiency (SE) criterion.

Given that precoding combined with power allocation has been found effective for improving the performance of multiuser (MU) multi-input multi-output (MIMO) communication systems, this technique has been thoroughly investigated in the past but mainly from a spectral efficiency (SE) point of view in a single cell scenario [4]-[6]. In the multi-cell context, interference from neighboring cells, i.e. other-cell interference $(\mathrm{OCI})$, has been shown to degrade the cell-edge user and overall SE of the system [7]. As a result, precoding/power allocation techniques for mitigating the effects of OCI have recently been proposed in [8], [9] and [10] but again only for optimizing the SE. Given that EE is growing in importance for designing future wireless communication systems, EE-based resource/power allocation is becoming a popular research topic. In the single-cell MU-MIMO setting, an EE optimal precoding/allocation algorithm for the multi-antenna broadcast channel has been proposed in [11], by adapting the work of [4] to EE requirement. Whereas, [12] has designed an EE-optimal resource allocation algorithm when orthogonality is used to remove intra-cell interference. In the multi-cell MU environment, the work of [13] has recently proposed an energy-efficient coordinated resource allocation method for the single antenna setting when perfect channel state information can be shared between cells.

In this paper, we propose a novel energy-efficient OCIaware precoding/power allocation for the downlink of multicell MU-MIMO systems by combining our SE-based precoding technique of [9] with our EE-based single-cell resource allocation algorithm of [12]. In comparison with [12], we here take into account interference from neighboring cells and assume that the base station (BS) as well as user equipments (UEs) have multiple antennas. Contrarily to [13], we only assume here statistical knowledge of the OCI and do not require any form of coordination between the BSs. The OCI noise covariance matrix of each user is fed back at the transmitter where it is incorporated in the precoding process, which is based on regularized channel inversion (RCI) [6], as in [9]. The power allocation is then designed for optimizing the EE of OCI-aware MU-MIMO systems when considering a realistic multi-antenna power consumption model with per-antenna power constraint; indeed in a realistic BS each transmit antenna has its own radio frequency (RF) chain and maximum transmit power [14]. In Section II, we first introduce both the system and power consumption models for the downlink of multi-cell MU-MIMO. Relying on these models, we then design in section III, an energy-efficient precoding/power allocation algorithm for the downlink of multi-cell MU-MIMO systems that is effective at mitigating the effects of OCI. In Section IV, we simulate the performances of our proposed scheme both in presence and absence of OCI and compare them against our SE-based RCI algorithm of [9]. Results first demonstrate the effectiveness of our approach for mitigating OCI, especially when the interference is strong and the number of UEs is large. In addition, our approach improves the EE of the system by making a better use of the transmit power, but at the cost of a lower SE, in comparison with the SE-based approach. Conclusions are finally drawn in Section V. 


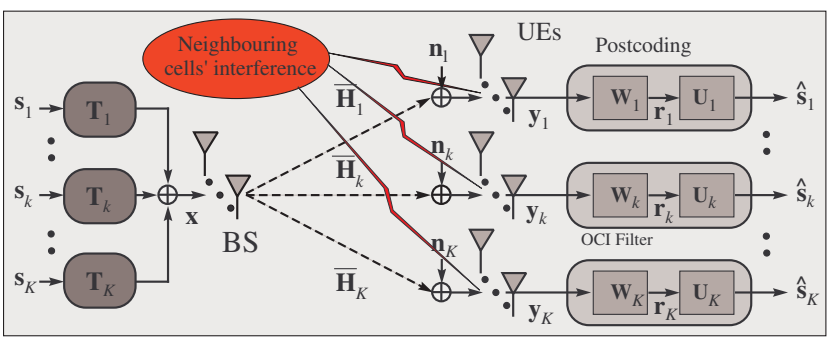

Fig. 1: Downlink of OCI-aware MU-MIMO system.

\section{Multi-Cell MU-Mimo System Description}

\section{A. System model}

In this paper, we study the $\mathrm{EE}$ in the downlink of a multi-cell MU-MIMO system, where a base station with $n$ transmit antennas broadcasts a signal $\mathbf{x}=\sum_{k=1}^{K} \mathbf{T}_{k} \mathbf{s}_{k}$ to $K$ user equipments (UEs) with $r_{k}$ antennas, as it is depicted in Fig. 1. Note that $\mathbf{T}_{k} \in \mathbb{C}^{n \times n}$ is the $k$-th user precoding matrix and $\mathbf{s}_{k} \in \mathbb{C}^{n \times 1}$, which we define as $\mathbf{s}_{k}=\left[\begin{array}{lll}\mathbf{0}^{1 \times \alpha_{k}} & \mathbf{s}_{k}^{\dagger} & \mathbf{0}^{1 \times\left(n-\alpha_{k+1}\right)}\end{array}\right]^{\dagger}$, where $\overline{\mathbf{s}}_{k} \in \mathbb{C}^{l_{k} \times 1}$ is the $k$-th message of length $l_{k}, \mathbf{0}^{1 \times \alpha_{k}}$ is an all zero vector of length $\alpha_{k}$ and $\alpha_{k}=\sum_{j=1}^{k-1} l_{j}$. Consequently, $\mathrm{E}\left\{\overline{\mathbf{s}}_{k} \overline{\mathbf{s}}_{k}^{\dagger}\right\}=\mathbf{I}_{l_{k}}$ and $\mathrm{E}\left\{\mathbf{x}^{\dagger} \mathbf{x}\right\}=P$, where $P$ is the total transmit power, $\mathbf{I}_{l_{k}}$ is a $l_{k} \times l_{k}$ identity matrix and $\mathrm{E}\{$.$\} stands for the expectation. In$ addition, we define $l$ as $l=\sum_{k=1}^{K} l_{k}$ and assume that $l_{k} \leq r_{k}$ and $l \leq n$. By considering that $n_{\mathrm{I}, k}$ effective interferers from neighbouring cells interfere with the transmit signal from the BS, the signal received at the $k$-th user can be expressed as

$$
\begin{aligned}
& \mathbf{y}_{k}=\overline{\mathbf{H}}_{k} \mathbf{x}+\mathbf{n}_{k}+\mathbf{n}_{\mathrm{I}, k} \\
& \mathbf{r}_{k}=\mathbf{H}_{k} \mathbf{x}+\mathbf{W}_{k}\left(\mathbf{n}_{k}+\mathbf{n}_{\mathrm{I}, k}\right)^{\prime}
\end{aligned}
$$

respectively, where $\mathbf{H}_{k}=\mathbf{W}_{k} \overline{\mathbf{H}}_{k}, \overline{\mathbf{H}}_{k} \in \mathbb{C}^{r_{k} \times n} \bmod -$ els the MIMO channel between the BS and the $k$-th user, $\mathbf{W}_{k} \in \mathbb{C}^{r_{k} \times r_{k}}$ is the $k$-th user OCI suppression filter, and $\mathbf{n}_{k} \in \mathbb{C}^{r_{k} \times 1}$ is a vector of independent zero-mean complex Gaussian noise entries with a variance of $\sigma_{k}^{2}$. In addition, $\mathbf{n}_{\mathrm{I}, k}=\mathbf{H}_{\mathrm{I}, k} \mathbf{x}_{\mathrm{I}, k}$ with $\mathbf{H}_{\mathrm{I}, k} \in \mathbb{C}^{r_{k} \times n_{\mathrm{I}, k}}$ and $\mathbf{x}_{\mathrm{I}, k} \in \mathbb{C}^{n_{\mathrm{I}, k} \times 1}$ being the $k$-th user MIMO OCI channel and OCI signal with $n_{\mathrm{I}, k}$ co-channel interferers from the neighboring cells, respectively. The OCI plus noise covariance matrix of user $k$ is such that [8]

$$
\mathbf{R}_{\mathbf{I}, k}=\sigma_{k}^{2} \mathbf{I}_{r_{k}}+\mathbf{H}_{\mathrm{I}, k} \mathbf{Q}_{\mathbf{I}, k} \mathbf{H}_{\mathrm{I}, k}^{\dagger},
$$

where $\mathbf{Q}_{\mathrm{I}, k}=\mathrm{E}\left\{\mathrm{x}_{\mathrm{I}, k} \mathbf{x}_{\mathrm{I}, k}^{\dagger}\right\}$ is the $k$-th user covariance matrix of interfering signals. We assume that each user $k$ can perfectly estimate its own OCI plus noise covariance matrix by using various techniques like those proposed in [15], [16], and then informs its serving BS by using the uplink feedback channel. Hence, $\mathbf{R}_{\mathrm{I}, k}$ is known at the BS and optimization is performed at the reference BS only, without need for any form of coordination between the interfering BSs.

According to equations (1) and (2), the $k$-th user downlink mutual information of the OCI-aware MU-MIMO system in
Fig. 1 can be given by

$$
\begin{aligned}
I\left(\mathbf{r}_{k}, \mathbf{s}_{k}\right)= & B \log _{2} \mid \mathbf{I}_{r_{k}}+\mathbf{H}_{k} \mathbf{T}_{k} \mathbf{T}_{k}^{\dagger} \mathbf{H}_{k}^{\dagger}\left(\mathbf{W}_{k} \mathbf{R}_{\mathrm{I}, k} \mathbf{W}_{k}^{\dagger},\right. \\
& \left.+\mathbf{H}_{k}\left(\mathbf{R}_{\mathbf{x}}-\mathbf{T}_{k} \mathbf{T}_{k}^{\dagger}\right) \mathbf{H}_{k}^{\dagger}\right)^{-1} \mid
\end{aligned}
$$

where $B$ is the channel bandwidth and $\mathbf{R}_{\mathbf{x}}=\sum_{j=1}^{K} \mathbf{T}_{j} \mathbf{T}_{j}^{\dagger}$ is the transmit covariance matrix. Note that $\mathbf{U}_{k}=$ $\mathbf{T}_{k}^{\dagger} \mathbf{H}_{k}^{\dagger}\left(\mathbf{H}_{k} \mathbf{R}_{\mathbf{x}} \mathbf{H}_{k}^{\dagger}+\mathbf{W}_{k} \mathbf{R}_{\mathbf{n}_{\mathrm{I}}, k} \mathbf{W}_{k}^{\dagger}\right)^{-1}$ when a linear Minimum Mean Squared Error receiver is utilized by each user [17].

\section{B. Power model and EE-SE trade-off formulation}

As far as the power consumptions of BS and UEs are concerning, it has been indicated in [14] and [18], respectively, that they can be modelled in a similar manner as $P_{\text {in }}=\Delta P+P^{C i}$. Given that each antenna has its own RF chain [14], this model has been refined for the MIMO setting in [19] as

$$
P_{\text {in }}=t\left(\Delta \bar{P}+P^{C i p A}\right)+P^{C i},
$$

where $\Delta$ is the RF dependent slope of the power model, $P^{C i p A}$ is the per-antenna circuit power, $t$ is the number of transmit antennas and $P^{C i}$ is the circuit power that is not dependent on $t$. In addition, the per-antenna transmit power, $\bar{P}$, is such that $\bar{P} \in\left[0, P_{n}^{\max }\right]$ with $P_{n}^{\max }$ being the per-antenna maximum transmit power. Consequently, the total consumed power in the downlink or uplink of a MU-MIMO system can be linearly abstracted as

$$
P_{\Sigma}=P_{\mathrm{c}}+\Delta P,
$$

when assuming the power model in (4) for the BS and UE(s), and where $P_{\mathrm{c}}=l P_{\mathrm{BS}}^{C i p A}+P_{\mathrm{BS}}^{C i}+\varsigma\left(\sum_{k=1}^{K} r_{k} P_{\mathrm{UE}}^{C i p A}+K P_{\mathrm{UE}}^{C i}\right)$ in the downlink direction. Moreover, $\varsigma$ characterizes the ratio between transmission and reception overhead powers with $0 \leq \varsigma \leq 1$. Intuitively, less overhead power is necessary for receiving than for transmitting signals.

Given that the energy efficiency (EE) can simply be defined as a ratio between the total consumed power and the sumrate [1], the downlink EE-SE trade-off of the OCI-aware MUMIMO system in Fig. 1 can be expressed as

$$
\begin{aligned}
E_{e} & \triangleq \frac{R_{\Sigma}=\sum_{k=1}^{K} I\left(\mathbf{r}_{k}, \mathbf{s}_{k}\right)}{P_{\Sigma}}=\frac{B}{P_{\mathbf{c}}+\Delta \operatorname{tr}\left\{\mathbf{R}_{\mathbf{x}}\right\}} \\
& \times \sum_{k=1}^{K} \log _{2}\left|\frac{\mathbf{W}_{k} \mathbf{R}_{\mathrm{I}, k} \mathbf{W}_{k}^{\dagger}+\mathbf{H}_{k} \mathbf{R}_{\mathbf{x}} \mathbf{H}_{k}^{\dagger}}{\mathbf{W}_{k} \mathbf{R}_{\mathrm{I}, k} \mathbf{W}_{k}^{\dagger}+\mathbf{H}_{k}\left(\mathbf{R}_{\mathbf{x}}-\mathbf{T}_{k} \mathbf{T}_{k}^{\dagger}\right) \mathbf{H}_{k}^{\dagger}}\right|,
\end{aligned}
$$

according to equations (3) and (5).

\section{ENERGY-EFFICIENT OCI-AWARE MU-MIMO PRECODING}

In this section, we propose to revisit the RCI algorithm of [6] in the downlink of OCI-aware multi-cell MU-MIMO systems by taking into account the OCI in the precoding process, as in our work of [9], and allocating power for optimizing the EE. As we mentioned earlier, the precoder only requires the OCI plus noise covariance matrix for each user for 
mitigating it. This approach is more practical than centralized coordination since each user can estimate its own covariance matrix without the need for multi-cell synchronization or training sequence. Given that $\mathbf{R}_{\mathrm{I}, k}$ in (2) is by definition a Hermitian positive definite matrix, $\forall k \in \mathcal{K}=\{1, \ldots, K\}$, it can be decomposed as $\mathbf{R}_{\mathrm{I}, k}=\mathbf{D}_{k}^{\dagger} \mathbf{D}_{k}$ by using Cholesky decomposition. By defining $\mathbf{W}_{k}=\sigma_{k} \mathbf{D}_{k}^{-1}$, the EE expression in can be expressed as $E_{e}=$

$$
\frac{B}{P_{\mathbf{c}}+\Delta \operatorname{tr}\left\{\mathbf{R}_{\mathbf{x}}\right\}} \sum_{k=1}^{K} \log _{2}\left|\frac{\sigma_{k}^{2} \mathbf{I}_{r_{k}}+\mathbf{H}_{k} \mathbf{R}_{\mathbf{x}} \mathbf{H}_{k}^{\dagger}}{\sigma_{k}^{2} \mathbf{I}_{r_{k}}+\mathbf{H}_{k}\left(\mathbf{R}_{\mathbf{x}}-\mathbf{T}_{k} \mathbf{T}_{k}^{\dagger}\right) \mathbf{H}_{k}^{\dagger}}\right| \text {. }
$$

By using $\mathbf{W}_{k}=\sigma_{k} \mathbf{D}_{k}^{-1}$ as the OCI mitigation filter, the OCI has been integrated with $\overline{\mathbf{H}}_{k}$ to form an equivalent channel matrix $\mathbf{H}_{k}$. Thus, each user feedbacks $\mathbf{H}_{k}$ in presence of OCI in place of $\overline{\mathbf{H}}_{k}$ when there is no OCI.

Next we modify the RCI precoding in [6] for cancelling intra-cell interference in an energy-efficient manner by defining the $k$-th user precoder at the $\mathrm{BS}, \mathbf{T}_{k}$, as

$$
\mathbf{T}_{k}=\mathbf{M}_{k} \mathbf{V}_{k} \mathbf{P}_{k},
$$

where $\mathbf{P}_{k}=\operatorname{diag}\left(\left[\sqrt{p_{k, 1}}, \ldots, \sqrt{p_{k, l_{k}}}\right]\right)$, for any $k \in \mathcal{K}$, and $\mathbf{V}_{k} \in \mathbb{C}^{l_{k} \times l_{k}}$ is the right singular matrix of $\mathbf{H}_{k} \mathbf{M}_{k}$. In addition, the columns of $\mathbf{M}_{k}$ are constrained to lie within the subspace of the vectors $\left\{\mathbf{t}_{k, 1}^{0}, \mathbf{t}_{k, 2}^{0}, \ldots, \mathbf{t}_{k, l_{k}}^{0}\right\}$ such that

$$
\mathbf{t}_{k, i}^{0}=\left(\sum_{\substack{j=1 \\ j \neq k}}^{K} \mathbf{H}_{j}^{\dagger} \mathbf{H}_{j}+\left(\mathbf{h}_{k, i}^{T}\right)^{\dagger} \mathbf{h}_{k, i}^{T}+\frac{l}{\gamma} \mathbf{I}_{n}\right)^{-1}\left(\mathbf{h}_{k, i}^{T}\right)^{\dagger},
$$

for any $i \in\left\{1, \ldots, l_{k}\right\}$, where $\mathbf{h}_{k, i}$ represents the $i$-th column of $\mathbf{H}_{k}^{T}$ and $\gamma$ is the average transmit SNR. By Denoting $\mathbf{m}_{k, i}$ as the $i$-th column of $\mathbf{M}_{k}$, vectors of the form $\left[\mathbf{m}_{k, 1}, \mathbf{m}_{k, 2}, \ldots, \mathbf{m}_{k, l_{k}}\right]$ are found as an arbitrary orthonormal basis of the vector space of vectors $\left[\mathbf{t}_{k, 1}^{0}, \mathbf{t}_{k, 2}^{0}, \ldots, \mathbf{t}_{k, l_{k}}^{0}\right]$ such that Gram-Schmidt orthogonalization can be utilized for obtaining the columns of $\mathbf{M}_{k}$ based on equation (9). Inserting $\mathbf{T}_{k}$ in (8) into (7), the latter can be reformulated as

$$
E_{e}(\mathbf{p})=\frac{B \sum_{k=1}^{K} \sum_{i=1}^{l_{k}} \log _{2}\left(1+p_{k, i} g_{k, i}\right)}{P_{\mathbf{c}}+\Delta \sum_{k=1}^{K} \sum_{i=1}^{l_{k}} p_{k, i}},
$$

where $\mathbf{p}=\left[p_{1,1}, \ldots, p_{1, l_{1}}, p_{2,1}, \ldots, p_{K, l_{K}}\right]$ and $g_{k, i}=$ $\frac{-1+\left\{\mathbf{G}_{k}\right\}_{i, i}}{p_{k, i}}$, for any $k \in \mathcal{K}, i \in\left\{1, \ldots, l_{k}\right\}$, with

$$
\mathbf{G}_{k}=\left(\mathbf{I}_{l_{k}}-\mathbf{T}_{k}^{\dagger} \mathbf{H}_{k}^{\dagger}\left[\mathbf{H}_{k} \mathbf{R}_{\mathbf{x}} \mathbf{H}_{k}^{-1}+\sigma_{k}^{2} \mathbf{I}_{r_{k}}\right]^{-1} \mathbf{H}_{k} \mathbf{T}_{k}\right)^{-1} \text {. }
$$

Given that each transmit antenna at the BS has its own power constraint [14], and each user uses $l_{k}$ transmit antenna to transmit its data, energy-efficient power allocation is then obtained by solving the following optimization problem

$$
\max _{\mathbf{p}} E_{e}(\mathbf{p}) \text {, s.t. } \mathbf{p} \succeq 0, p_{k, i} \leq P_{u}^{\max }, \forall u \in\{1, \ldots, l\},
$$

where $u=\sum_{j=1}^{k-1} l_{j}+i$. This problem being similar to the convex problem in [12], the per-antenna optimal transmit

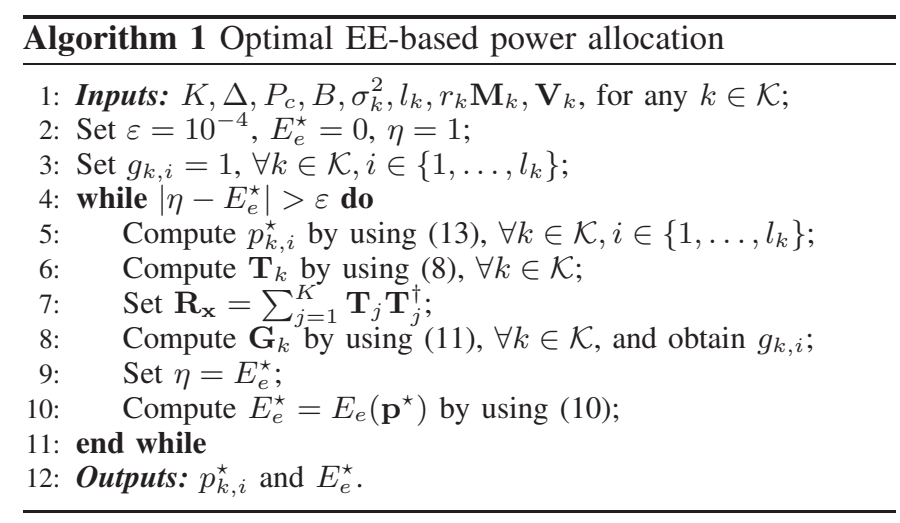

TABLE I: Power parameter values

\begin{tabular}{|c||c|c|c|c|}
\hline Parameters & $\Delta$ & $P^{C i p A}(\mathrm{~W})$ & $P^{C i}(\mathrm{~W})$ & $P_{u}^{\max }(\mathrm{W})$ \\
\hline BS & $4.7[14]$ & 100 & 180 & $20[14]$ \\
\hline UE & - & 0.03 & 0.07 & - \\
\hline
\end{tabular}

power can accordingly be expressed as

$$
p_{k, i}^{\star}=\left[\frac{B}{\ln (2) \Delta E_{e}^{\star}}-g_{k, i}^{-1}\right]_{0}^{P_{u}^{\max }}
$$

such that $0 \leq p_{k, i}^{\star} \leq P_{u}^{\max }, \forall k \in \mathcal{K}, i \in\left\{1, \ldots, l_{k}\right\}$, and where $E_{e}^{\star}=E_{e}\left(\mathbf{p}^{\star}\right)$ is the optimal unconstrained EE. By inserting $p_{k, i}^{\star}$ in (13) into (10) and (11), the power allocation problem in (12) reverts to a single variable problem, which can be straightforwardly solved by using our algorithm in Algorithm 1.

\section{Numerical Results AND Discussions}

In this section, we compare the performances of our proposed algorithm for improving the EE in OCI-aware MUMIMO systems against our SE-based approach of [9] in terms of transmit power, SE and EE when OCI occurs. In Fig. 2, we also plot EE-based precoding/power allocation results in absence of OCI for benchmarking purpose.

In the following, we assume as in [8] that $\mathbf{Q}_{\mathrm{I}, k}=$ $\left(P_{\mathrm{I}, k} / n_{\mathrm{I}, k}\right) \mathbf{I}_{n_{\mathrm{I}, k}}$, where $P_{\mathrm{I}, k}$ is the power of the $k-t h$ interference signal. Even though this is not a typical assumption, it has proved useful in [8] for evaluating the degradation due to OCI in the worst case scenario. In addition, we set $\gamma_{\mathrm{I}, k}=P_{\mathrm{I}, k} / \sigma_{k}^{2}$ as the interference to noise ratio (INR), where $\sigma_{2, k}^{2}=\sigma^{2}$ for any $k \in \mathcal{K}$. As far as the MU-MIMO channel is concerned, we assume a single-tap i.i.d. Rayleigh fading channel between the BS and any UEs. Moreover, we set $B=1, \varsigma=1 / 2$, and the power parameters $\Delta, P^{C i p A}, P^{C i}$ as well as $P_{u}^{\max }$ based on Table I. We also considered $5 \times 10^{3}$ realizations of each channel matrix for evaluating the performances of the different schemes via a Monte-Carlo process.

In Fig. 2, we first compare the performance of our energyefficient OCI-aware scheme (proposed scheme) in absence and presence of OCI for $n=l=8, P_{u}^{\max }=20 \mathrm{~W}$, $\forall u \in\{1, \ldots, l\}$, and different noise to interference levels as well as numbers of co-channel interferers. In the lower and upper parts of Fig. 2, $K=2, r_{k}=l_{k}=4$ and $K=4$, 


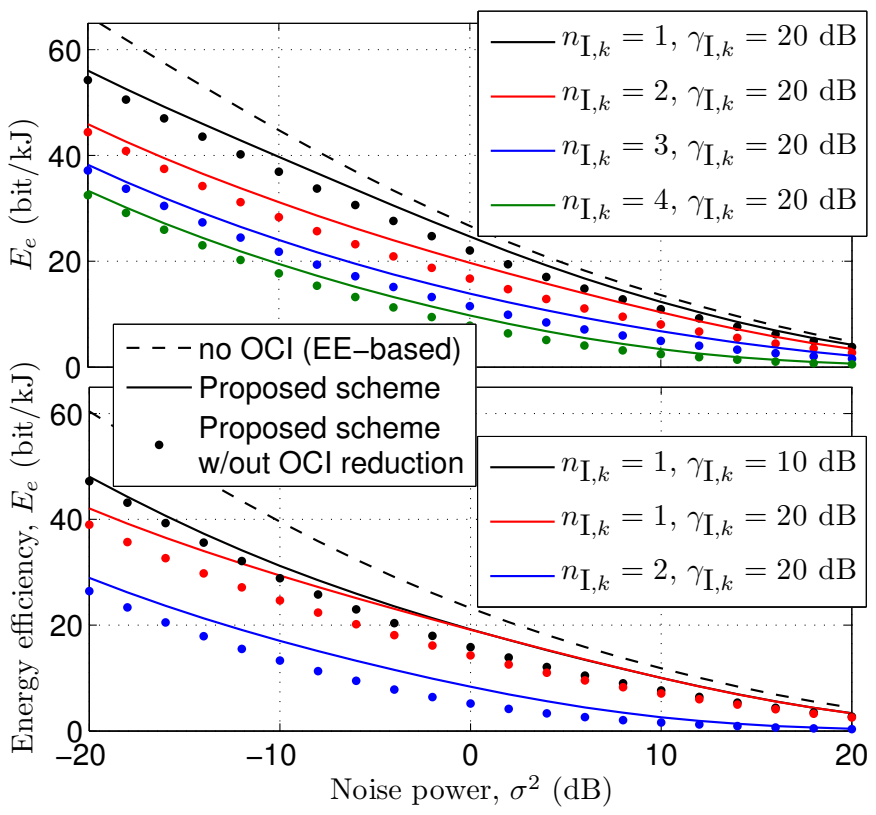

Fig. 2: Comparison of our energy-efficient OCI-aware scheme in absence and presence of OCI for $n=l=8, P_{u}^{\max }=20$ $\mathrm{W}, \forall u \in\{1, \ldots, l\}$, and various $n_{\mathrm{I}, k}$ and $\gamma_{\mathrm{I}, k}$ values.

$r_{k}=l_{k}=2$, for any $k \in \mathcal{K}$, respectively. Comparing the results for different numbers of co-channel interferers in the upper part of Fig. 2 indicates that when $n_{\mathrm{I}, k}$ increases then the EE decreases, which is in-line with the SE-based results of [9]. The graph also shows that our proposed scheme is more effective at mitigating the effects of OCI when $n_{\mathrm{I}, k}$ is low, i.e. the difference between the plain (OCI-aware) and dotted (no OCI reduction) lines are larger; indeed, when $n_{\mathrm{I}, k}$ is low, there is more degree of freedom for mitigating the OCI. Whereas, the lower part of Fig. 2 indicates that the stronger the INR is, the worst the EE performance is, but the more energy can be saved by using our scheme; the gap between the plain and dotted lines increases with $\gamma_{\mathrm{I}, k}$. When comparing the upper with the lower part of Fig. 2 for $n_{\mathrm{I}, k}=1$ and $\gamma_{\mathrm{I}, k}=20 \mathrm{~dB}$, it also reveals that the effectiveness our scheme for mitigating $\mathrm{OCI}$ also increases with the number of users.

In Fig. 3, we compare the performance of our proposed scheme against the SE-based approach of [9] in presence of $\mathrm{OCI}$ as a function of the per-antenna maximum transmit power, $P_{u}^{\max }$, for $n=l=8, K=4, \sigma^{2}=0 \mathrm{~dB}, r_{k}=l_{k}=2$, $n_{\mathrm{I}, k}=1$ and $\gamma_{\mathrm{I}, k}=10 \mathrm{~dB}$, for any $k \in \mathcal{K}$. The results show that our proposed approach can drastically reduce (up to $60 \%$ ) the per-antenna consumed power in comparison with the SE-based approach, especially if $P_{u}^{\max }$ is large; however, our approach power savings will come at the cost of a reduce SE (down to 20\%). Overall, our proposed EE-based scheme can help to improve the EE by up to $15 \%$ in comparison with the SE-based scheme, in this particular scenario. Moreover, when comparing the OCI-aware with the no OCI reduction $E_{e}$ results, it indicates that $\mathrm{OCI}$ filtering is also very beneficial for saving energy, a saving of $20 \%$ on average.

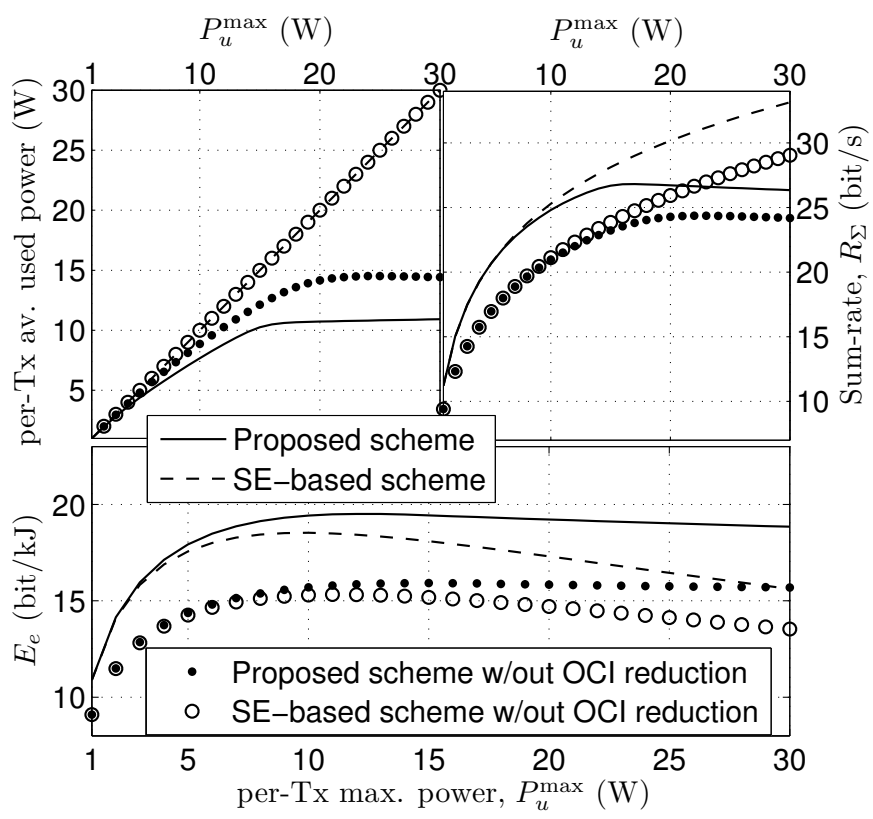

Fig. 3: Comparison of our energy-efficient OCI-aware scheme against the SE-based approach of [9] in terms of per-antenna transmit power, sum-rate and EE for $n=l=8, K=4$, $\sigma^{2}=0 \mathrm{~dB}, r_{k}=l_{k}=2, n_{\mathrm{I}, k}=1$ and $\gamma_{\mathrm{I}, k}=10 \mathrm{~dB}, \forall k \in \mathcal{K}$.

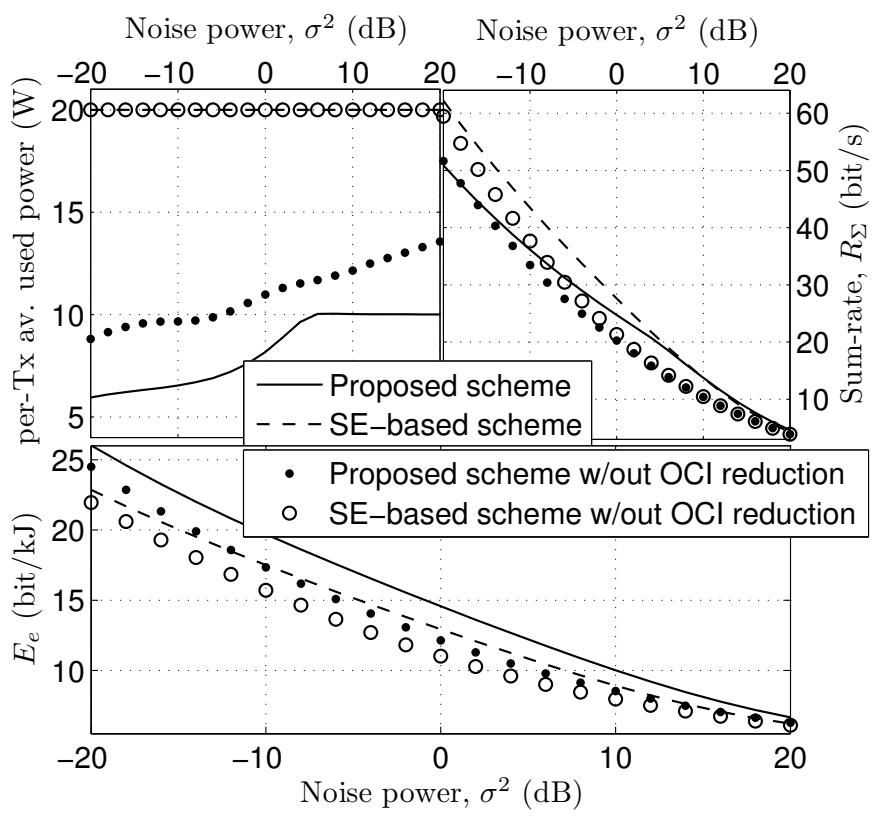

Fig. 4: Comparison of our energy-efficient OCI-aware scheme against the SE-based approach of [9] in terms of per-antenna transmit power, sum-rate and EE for $n=l=8, K=4$, $P_{u}^{\max }=20 \mathrm{~W}, \forall u \in\{1, \ldots, l\}, r_{k}=l_{k}=2, n_{\mathrm{I}, k}=1$ and $\gamma_{\mathrm{I}, k}=20 \mathrm{~dB}, \forall k \in \mathcal{K}$.

In Fig. 4, we compare the performance of our proposed scheme against the SE-based approach of [9] in presence of OCI as a function of the noise power, $\sigma^{2}$, for the same parameters as Fig. 3, except that $\gamma_{\mathrm{I}, k}=20 \mathrm{~dB}, \forall k \in \mathcal{K}$ and 
$P_{u}^{\max }=20 \mathrm{~W}, \forall u \in\{1, \ldots, l\}$. The results again show the effectiveness of our approach for saving transmit power and improving the EE in comparison with the SE-based approach. It also further highlights the effectiveness of our approach for mitigating the effects of OCI and saving extra energy.

\section{CONClusion}

In this paper, we have designed a novel energy-efficient OCI-aware precoding/power allocation algorithm for the downlink of multi-cell MU-MIMO systems by relying on RCI and considering a realistic multi-antenna power consumption model. In our algorithm, the precoder is designed to mitigate at the same time the OCI and intra-cell interference while the power allocation is performed on a per-antenna basis for optimizing the EE of multi-cell MU-MIMO systems. We have simulated the performances of our proposed scheme both in presence and absence of OCI and have compared them against a classic SE-based RCI algorithm. Results have demonstrated the effectiveness of our approach for mitigating OCI, especially when the interference is strong and the number of UEs is large. Moreover, they have shown that our approach improves the EE of the system by saving transmit power but at the cost of a lower SE in comparison with the SE-based approach. In the future, we plan to design a similar method but for the uplink of OCI-aware MU-MIMO systems.

\section{ACKNOWLEDGMENT}

The research leading to these results has received funding from the European Commission's Seventh Framework Programme FP7/2007-2013 under grant agreement n³18273project LEXNET.

\section{REFERENCES}

[1] S. Verdu, "Spectral Efficiency in the Wideband Regime," IEEE Trans. Inf. Theory, vol. 48, no. 6, pp. 1319-1343, Jun. 2002.

[2] H. M. Kwon and T. G. Birdsall, "Channel Capacity in Bits per Joule," IEEE J. Ocean. Eng., vol. OE-11, no. 1, pp. 97-99, Jan. 1986.

[3] V. Rodoplu and T. H. Meng, "Bits-per-Joule Capacity of Energy-limited Wireless Networks," IEEE Trans. Wireless Commun., vol. 6, no. 3, pp. 857-865, Mar. 2007.
[4] N. Jindal, W. Rhee, S. Vishwanath, S. A. Jafar, and A. Goldsmith, "Sum Power Iterative Water-Filling for Multi-Antenna Gaussian Broadcast Channels," IEEE Trans. Inf. Theory, vol. 51, no. 4, pp. 1570-1580, Apr. 2005.

[5] Q. H. Spencer, A. L. Swindlehurst, and M. Haardt, "Zero-Forcing Methods for Downlink Spatial Multiplexing in Multiuser MIMO Channels," IEEE Trans. Signal Process., vol. 52, no. 2, pp. 461-471, Feb. 2004.

[6] H. Lee, K. Lee, B. M. Hochwald, and I. Lee, "Regularized Channel Inversion for Multiple-Antenna Users in Multiuser MIMO Downlink," in Proc. IEEE ICC'08, Beijing, China, May 2009.

[7] O. Somekh, B. M. Zaidel, and S. Shamai, "Sum Rate Characterization of Joint Multiple Cell-site Processing," IEEE Trans. Inf. Theory, vol. 53, no. 12, pp. 4473-4497, Dec. 2007.

[8] S. Shim, J. S. Kwak, R. W. Heath, and J. G. Andrews, "Block Diagonalization for Multi-User MIMO with Other-Cell Interference," IEEE Trans. Wireless Commun., vol. 7, no. 7, pp. 2671-2681, Jul. 2008

[9] Y. Sambo, F. Héliot, and M. A. Imran, "An interference-aware precoding scheme for the downlink of multi-cell multi-user MIMO communication," in Proc. IEEE ICAST, Kumasi, Ghana, Oct. 2012.

[10] S. Zhao, Y. Zhao, H. Sun, J. Liu, L. Zhang, and L. Gui, "An interferenceaware precoding scheme with other-cell interference for downlink multiuser MIMO channel," in Proc. IEEE VTC-Fall, Ottawa, Canada, Sep. 2010.

[11] J. Xu, L. Qiu, and C. Yu, "Improving Energy Efficiency Through Multimode Transmission in the Downlink MIMO Systems," EURASIP J. Wireless Commun. and Net., vol. 2011, no. 200, Dec. 2011.

[12] F. Héliot, M. A. Imran, and R. Tafazolli, "Energy-efficiency based resource allocation for the orthogonal multi-user channel," in Proc. IEEE VTC-Fall, Québec city, Canada, Sep. 2012.

[13] — "Low-complexity energy-efficient coordinated resource allocation in cellular systems," IEEE Trans. Commun., vol. 61, no. 6, pp. 22712281, Jun. 2013.

[14] G. Auer et al., "How Much Energy is Needed to Run a Wireless Network ?" IEEE Wireless Commun., vol. 18, no. 5, pp. 40-49, Oct. 2011

[15] M. L. Honig, U. Madhow, and S. Verdu, "Blind adaptive multiuser detection," IEEE Trans. Inf. Theory, vol. 41, no. 7, pp. 944-960, Jul. 1995.

[16] A. Kansal, S. N. Batalama, and D. A. Pados, "Adaptive maximum SINR rake filtering for DS-CDMA multipath fading channels," IEEE J. Sel. Areas Commun., vol. 16, no. 12, pp. 1765-1773, Dec. 1998.

[17] A. J. Tenenbaum and R. S. Adve, "Joint Multiuser Transmit-Receive Optimization Using Linear Processing," in Proc. IEEE ICC '04, Paris, France, Jun. 2004, pp. 588-592.

[18] G. Miao, N. Himayat, and G. Y. Li, "Energy-Efficient Link Adaptation in Frequency-Selective Channels," IEEE Trans. Commun., vol. 58, no. 2, pp. 545-554, Feb. 2010.

[19] F. Héliot, M. A. Imran, and R. Tafazolli, "On the energy efficiencyspectral efficiency trade-off over the MIMO Rayleigh fading channel," IEEE Trans. Commun., vol. 60, no. 5, pp. 1345-1356, May 2012. 